Introduction: Ghrelin has been shown to decrease insulin levels and increase appetite, body weight gain and fat accumulation. It is decreased in obesity and associated diseases. Obestatin has been isolated as its counterpart, with the common precursor, prepropeptid. Morbid obesity has also been associated with tryptophan metabolism, as 5-hydroxytryptophan, serotonin, is involved in regulation of satiety. We aimed to assess possible correlations between tryptophan degradation and serum ghrelin/ obestatin and metabolic parameters in obese children and adolescents and non-obese controls.

Method: Serum ghrelin, obestatin (RIA, Phoenix, Germany), fasting and postprandial (oral glucose tolerance test) insulin, neopterin (ELISA, Brahms Hennigsdorf), tryptophan, kynurenine (HPLC) in 356 overweight and obese (mean age 11.3 ( $\mathrm{sD} 2.97$ ) years; $\mathrm{f}=\mathrm{m}$ ), otherwise healthy children and thirty-two non-obese controls.
Results: No difference was found in ghrelin serum levels between obese and non-obese controls. With the exception of ghrelin all investigated parameters differ significantly as compared with the obese group. Furthermore we found a significant association of ghrelin with weight and BMI, but not with obestatin. Tryptophan degradation significantly correlated with ghrelin, but not with obestatin. In addition, tryptophan correlated significantly with parameters of the metabolic syndrome.

Conclusions: Our results suggest a strong negative correlation of ghrelin and weight/BMI in obese children and adolescents. Furthermore, we found a significant correlation between tryptophan degradation rate and ghrelin suggesting central biochemical collaterals. Thus, ghrelin may play a role in mood disturbances, which in turn ultimately lead to increased energy intake.

\title{
Obese children with normal insulin sensitivity respond differently from those with insulin resistance to lifestyle modifications
}

\author{
L Gilardini, L Pasqualinotto, M Croci, L Vallone and C Invitti \\ Department of Medical Sciences and Rehabilitation, Istituto Auxologico Italiano, Milan, Italy
}

Background: Insulin resistance is considered the unifying mechanism for the derangements associated with metabolic syndrome and its related diseases and is a frequent feature of childhood obesity. Lifestyle intervention is recommended to treat insulin resistance. Recently it has been reported that weight loss induced by diet and exercise, paradoxically increases health risk in metabolically healthy individuals. This finding raised the doubt that uncomplicated obesity would not benefit from lifestyle-induced weight loss.

The aim of our study was to assess the effects of a 3-month lifestyle intervention on insulin sensitivity and its related variables (blood pressure, HDL-cholesterol, triglycerides, fasting and $2 \mathrm{~h}$ post-OGTT glucose, ALT, CRP, white blood cells) in 202 obese children (36.4\% prepubertal, BMI Z-score $3 \cdot 2$ (SD 0.7)). In particular the study analysed if a short-term lifestyle intervention may have detrimental effects on metabolic variables in young people with normal insulin sensitivity. Lifestyle intervention consisted in (i) mild caloric restriction with an appropriate protein intake according to the Italian recommendations for age, and $0.5 \mathrm{~g} / \mathrm{kg}$ of ideal body weight of soluble and insoluble fibres, $25 \%$ of fat $(10 \%$ saturated fat $)$ and $55-65 \%$ of carbohydrates (45-55\% of complex sugars and $10 \%$ of simple sugars, (ii) one-hour weekly session at the gym dedicated to the education to the physical activity under the guidance of trainer +240 min of physical activity at home during the week, (iii) family psychological counselling and psycho-educational groups. HOMA-IR was used as a surrogate marker of liver insulin resistance and the OGTT-derived Matzuda index as a marker of muscular insulin sensitivity. Metabolically healthy obese (MHO) was defined by normal values of blood pressure, lipids and glucose.

Results: In the whole cohort, the 3-month lifestyle intervention induced a significant decrease in weight, waist circumference, fat mass and ALT levels with an increase in fat-free mass. However, it was possible to distinguish two groups of subjects, one in which insulin sensitivity improved (Group 1) at the end of the intervention and one in which it worsened (Group 2 represented by $45 \%$ of subjects). Before the intervention, Group 1 and Group 2 subjects were comparable in BMI, waist circumference, body composition, \% of pre-pubertal children and males, family history of diabetes, dietary intake and heart rate and \% MHO subjects. Group 1 children were more insulin resistant (insulin $16 \cdot 2(\mathrm{SD} 7 \cdot 1) \mathrm{mU} / \mathrm{ml} v$. $10 \cdot 4(\mathrm{sD} 5 \cdot 3) \mathrm{mU} / \mathrm{ml}, P<0 \cdot 0001$; HOMA-IR $3 \cdot 4(\mathrm{sD} 1 \cdot 6) v$. 
$2 \cdot 1$ (SD 1·1), $P<0 \cdot 0001$; Matsuda index (4.7 (sD 2.7) $v \cdot 6 \cdot 7$ (SD 3.9), $P<0 \cdot 001$ ) and had higher glucose levels (fasting glucose $85 \cdot 4$ (sD 6.6) mg/dl $v .82 \cdot 4(\mathrm{sD} 5 \cdot 8$ ) $\mathrm{mg} / \mathrm{dl}, P<0 \cdot 01$; $2 \mathrm{~h}$ glucose $91 \cdot 8$ (sD $15 \cdot 8) \mathrm{mg} / \mathrm{dl} v .85 \cdot 2$ (SD 14.6) $\mathrm{mg} / \mathrm{dl}$, $P<0.05$ and higher) than those of Group 2. Lifestyle intervention induced a comparable significant decrease in weight, waist circumference, fat mass, heart rate and ALT (and increase in fat-free mass) in the two groups. Unlike Group 1 who had a significant decrease in glucose and triglyceride levels, Group 2 had a significant increase in glucose and white blood cells values and triglycerides (NS) ( $\mathrm{s} \%$ statistically significant between groups).

Conclusions: (i) Insulin resistance is often not associated with metabolic alterations in young obese people; (ii) lifestyle intervention, at least in a short-term period, induces metabolic improvements when obese children are insulin resistant whereas it may paradoxically worsen insulin sensitivity, glucose tolerance and inflammation when they are insulin sensitive; (iii) this finding is similar to that described in adult obese and its path physiology remains to be clarified.

\title{
Evaluation of new obesity-related individual metabolic and vascular risk profiles: the STYJOBS/EDECTA resource
}

\author{
Verena Biedermann ${ }^{1}$, Gunter Almer ${ }^{1}$, Sieglinde Zelzer ${ }^{1}$, Carmen Bauer-Denk $^{1}$, \\ Andreas Meinitzer ${ }^{1}$, Florian Freytag ${ }^{1}$, Renate Horejsi ${ }^{1}$, Reinhard Möller ${ }^{1}$, \\ Wolfgang Schnedl ', Kerstin Hingerl' ${ }^{1}$, Karl Koschutnig ${ }^{1}$, Gernot Reishofer ${ }^{1}$, \\ Renate Kruschitz ${ }^{1}$, Marcel Scheideler ${ }^{1}$, Martie Truschnig-Wilders ${ }^{1}$, Sandra Wallner- \\ Liebmann ${ }^{1}$, Robert Gasser ${ }^{1}$, Gerwin Bernhardt ${ }^{1}$, Elisabeth Kraigher-Krainer ${ }^{1}$, \\ Harald Mangge ${ }^{1}$, Daniel Weghuber ${ }^{2}$, Sabine Ebner ${ }^{2}$, Barbara Kofler ${ }^{2}$, Waltraud Eder ${ }^{2}$, \\ Wolfgang Sperl ${ }^{2}$, Elisabeth Ardelt-Gattinger ${ }^{3}$, Susanne Ring-Dimitriou ${ }^{3}$, \\ Monika Siegrist ${ }^{4}$, Christina Holzapfel ${ }^{4}$, Martin Halle ${ }^{4}$, Helmut Langhof $^{5}$ and \\ Vasan S Ramachandran ${ }^{6}$ \\ ${ }^{1}$ Medical University of Graz, Austria: ${ }^{2}$ Paracelsus Private University, Salzburg, Austria, Salzburg University, \\ Salzburg, Austria: ${ }^{3}$ Technical University Munich, Munich, Germany: ${ }^{4}$ Obesity Rehabilitation Clinic Schönsicht, \\ Berchtesgaden, Germany: ${ }^{5}$ Boston University School of Medicine, The Framingham Heart Study, Boston, MA, USA: \\ ${ }^{6}$ Obesity Academy Austria (OAA), Austria
}

Obesity is dramatically increasing in the western world. Atherosclerosis and type 2 diabetes, major consequences of obesity, start early in life and result in potentially fatal CVD. The STYrian Juvenile OBesity Study (STYJOBS) is a prospective study to improve the understanding of the major pathologic sequels of obesity by investigation of the 'non-biased' early phase in juveniles. EDECTA (Early DeteCTion of Atherosclerosis) extends STYJOBS to young and middle aged adults up to the age of 55 years.

Aims: Identification of 'individual metabolic high-risk patterns' in obesity by linking lab parameters (e.g. adipokines, lipid subfractions, natriuretic peptides, oxidative 'stress' biomarkers, molecular genetics, epigenetics), individual adipose tissue topography (lipometry), early vascular changes (carotids sonography), lifestyle habits, sport performance diagnostics and clinical data. STYJOBS/EDECTA-Database.

To establish a large serum/plasma/DNA resource of obese probands, their parents, and normal weight age matched controls comprehending the whole preclinical phase from 0 to 55 years (juveniles and adults) for advanced risk research (e.g. molecular genetics, epigenetics, microRNAs) in obesity. STYJOBS/EDECTA-Bioresource. To improve the understanding of addiction-like behaviour in obesity (e.g. imaging by 3 Tesla NMR after visual stimulation, analysis of dopamine receptor expression, link insulin resistance - control of hedonic inputs, craving). To establish by these data new strategies for prevention of lifestyle-associated diseases. STYJOBS/ EDECTA-Prevention.

Currently, STYJOBS/EDECTA comprehends data and biomaterial of $\sim 900$ participants aged between 3 and 55 years. The cohort is extended up to an end size of $n 1600$ to achieve one of the largest data and biomaterial resources addressing the preclinical phase of major sequels of obesity such as type 2 diabetes, myocardial infarction, stroke and cancer. These data should give a new basis for an improved personalised prevention. A broad international interdisciplinary co-operational network of scientists is involved in the STYJOBS/EDECTA activities. 